\title{
Modelo didáctico para matemáticas básicas en el programa de ingeniería química
}

\author{
Didactic model for basic mathematics in the chemical engineering program
}

GARCÍA-NAVARRO, Norma †*, BAUZA-MENDOZA, Guadalupe, ROJAS-HERNÁNDEZ, Miguel Angel y RAMOS-GONZÁLEZ, Luz María

Universidad Veracruzana, Facultad de Ingeniería Química.

ID $1^{\text {er }}$ Autor: Norma, García-Navarro / ORC ID: 0000-0002-0381-6617, Researcher ID Thomson: G-9585-2018, CVU CONACYT ID: 49611

ID $1^{\text {er }}$ Coautor: Guadalupe, Bauza-Mendoza / ORC ID: 0000-0001-8079-0812, Researcher ID Thomson: I-6638-2018, CVU CONACYT ID: 697137

ID 2do Coautor: Miguel Angel, Rojas-Hernández / ORC ID: 0000-0001-9294-5842

ID 3er Coautor: Luz María, Ramos-González / ORC ID: 0000-0002-8658-2472, Researcher ID Thomson: G-1385-2019, CVU CONACYT ID: 972621

DOI: $10.35429 / J H S .2019 .10 .3 .21 .29$

Recibido 15 de Octubre, 2019, Aceptado, 02 de Diciembre, 2019

\begin{abstract}
Resumen
Los problemas del aprendizaje de las matemáticas constituyen un campo particularmente importante en la relación educativa. En cualquier ingeniería y específicamente en Ingeniería Química (IQ) se requiere de una serie de habilidades matemáticas para el aprendizaje y resolución de problemas. En virtud de haber realizado una revisión sobre los índices de reprobación en las experiencias educativas del programa de IQ, se detectaron que los valores más altos eran precisamente las matemáticas básicas, esto llevó a plantear la solución proponiendo un cambio en la enseñanza con el objetivo de mejorar el aprendizaje y con ello, reducir los índices de reprobación a partir del uso de un modelo didáctico. El modelo didáctico se basa en 3 etapas que abarcan información bibliográfica, documental y estadística, para la parte de intervención, enmarcado dentro de la modalidad investigación cuantitativa de campo de tipo descriptivo, con características de observación participante. Al implementarse, permitió observar algunos cambios significativos en referencia a los hábitos de estudio y actitud que presentan los alumnos frente a las matemáticas y sobre los estilos adoptados por los profesores para esta cátedra, con lo que finalmente se evaluó concluyéndose que efectivamente mejora los índices de aprobación y el aprendizaje significativo.
\end{abstract}

Matemáticas, Aprendizaje, Ingeniería

\begin{abstract}
The problems of learning mathematics are a particularly important field in the educational relationship. In the degree of any engineering and specifically in Chemical Engineering (IQ) requires a series of mathematical skills for learning and problem solving. By virtue of having made a revision on the indices of failure in the educational experiences of the IQ program, it was detected that the highest values were precisely the basic mathematics, this led to propose the solution proposing a change in the teaching with the objective of improve learning and with it, reduce failure rates from the use of a didactic model. The didactic model is based on 3 stages that include bibliographic, documentary and statistical information, for the intervention part, framed within the quantitative field research modality of descriptive type, with characteristics of participant observation. When implemented, it allowed to observe some significant changes in reference to the habits of study and attitude that the students present before the mathematics and on the styles adopted by the professors for this chair, with which finally it was evaluated concluding that it effectively improves the indexes of approval and meaningful learning.
\end{abstract}

Mathematics, Learning, Engineering

Citación: GARCÍA-NAVARRO, Norma, BAUZA-MENDOZA, Guadalupe, ROJAS-HERNÁNDEZ, Miguel Angel y RAMOS-GONZÁLEZ, Luz María. Modelo didáctico para matemáticas básicas en el programa de ingeniería química. 2019 3-10: $21-29$

\footnotetext{
* Correspondencia del Autor (Correo electrónico: nogarcia@uv.mx)

$\dagger$ Investigador contribuyendo como primer autor.
} 


\section{Introducción}

Independientemente de la etapa educativa en la que ejerza su profesión, todo profesor, enfoca y realiza su labor docente partiendo de una serie de creencias, decisiones y consideraciones en relación a lo que significa enseñar y cómo sus alumnos adquieren los conocimientos de una manera adecuada para obtener mejores resultados. Estas ideas, la mayoría sustentadas en la experiencia personal de cada profesor, influyen de manera directa sobre la construcción del conocimiento en los estudiantes, y lo que es más importante, en la visión que los mismos vayan formándose de cada asignatura.

La matemática es una manera de pensar que se utiliza para resolver diversos problemas que se nos plantean en nuestra vida cotidiana, un modo de razonar; es un campo de exploración, investigación e invención en el cual se descubren nuevas ideas cada día. Ha estado presente desde el principio de los tiempos y ha sido necesaria para desarrollar procesos y actividades, de forma simple o compleja, a lo largo de toda nuestra vida, pues desde pequeños estamos en contacto con las formas y los números, nos ubicamos en el espacio, clasificamos, contamos, realizamos multitud de procesos y desarrollamos múltiples destrezas y capacidades en relación a la matemática a través de ese afán innato de descubrir; sin embargo, existen grandes dificultades para lograr conectar el trabajo educativo con un interés intrínseco de los estudiantes respecto al saber matemático, lo cual orilla a los profesores a promover una serie de "estímulos" externos, a la tarea propiamente matemática que pueden consistir en un incentivo al estudiante o en acciones que tienen un papel sancionador.

Por una parte, las matemáticas aparecen como algo alejado de la realidad; por la otra, los índices de bajo aprovechamiento e incluso de reprobación parecen alarmantes en las escuelas. Esta es quizá una de las cuestiones nodales en la educación de las matemáticas. Bajo este escenario se realizó una revisión sobre los índices de reprobación en las experiencias educativas del programa de Ingeniería Química (IQ), detectando que los valores más altos correspondían a las matemáticas básicas y que en las entrevistas a profesores, había coincidencia en que la problemática estaba en la forma de enseñanza, carente de estrategias de aprendizaje.
Todo lo anterior llevó a preguntarse ¿de qué forma se puede mejorar el aprendizaje en una asignatura tan importante en la Ingeniería Química? Para dar respuesta se propuso realizar un diagnóstico de la problemática, mediante verificaciones diarias de campo y video para el registro $\mathrm{y}$ análisis de resultados; $\mathrm{y}$, posteriormente, plantear posibles soluciones que permitan disminuir los índices de reprobación.

Con esta propuesta se pretende mejorar el aprendizaje de matemáticas básicas en IQ con la finalidad de reducir los índices de reprobación a partir del uso de un modelo didáctico. Este modelo siguió el estilo de la investigación cuantitativa de campo de tipo descriptivo, con características de observación participante que siguió con una propuesta de un modelo didáctico para mejorar el aprendizaje en Matemáticas Básicas y que finalmente se evaluó para determinar su efectividad.

\section{Marco Conceptual}

\section{Didáctica de matemáticas}

La didáctica de las matemáticas centra su interés en todos aquellos aspectos que forman parte del proceso de enseñanza de este campo de conocimiento, apoyando a los profesores con herramientas necesarias para impartir la docencia sobre unos cimientos consistentes, orientándole y guiándole en el ejercicio de su profesión en beneficio del aprendizaje de sus alumnos.

Entre los principales desafíos de los diferentes modelos o enfoques educativos, se encuentra el proceso de enseñanza y evaluación de matemáticas. En este sentido, existen diversas perspectivas sobre el aprendizaje de matemáticas, que han derivado en diferentes posturas sobre la didáctica que se debe seguir en esta ciencia. (Ruiz, 2006) resalta la importancia del grupo de investigadores franceses que conforman la Didactique des mathématiques como la corriente que ha buscado establecer los métodos, procesos de investigación y núcleo teórico propio de la didáctica de las matemáticas.

La evolución de la didáctica de las matemáticas se ha desarrollado en diferentes etapas, en la Tabla 1 se presenta de forma sintetizada. 


\begin{tabular}{|l|l|}
\hline Etapa & \multicolumn{1}{|c|}{ Caracterización } \\
\hline Antigua & $\begin{array}{l}\text { Es un arte, difícilmente susceptible } \\
\text { de ser analizada, controlada y } \\
\text { sometida a reglas, depende del } \\
\text { profesor y su habilidad para } \\
\text { enseñarlas. }\end{array}$ \\
\hline Clásica & $\begin{array}{l}\text { Considera el aprendizaje como un } \\
\text { proceso psico-cognitivo fuertemente } \\
\text { influenciado por factores } \\
\text { motivacionales, afectivos y sociales } \\
\text { que permiten resolver los obstáculos } \\
\text { que enfrenta el profesor o el alumno. }\end{array}$ \\
\hline $\begin{array}{l}\text { Didáctica } \\
\text { fundamental }\end{array}$ & $\begin{array}{l}\text { Se plantea el uso de modelos de la } \\
\text { actividad matemática así como del } \\
\text { proceso de enseñanza aprendizaje } \\
\text { para resolver problemas didácticos } \\
\text { como el papel de los algorítmos, la } \\
\text { creatividad y la resolución de } \\
\text { problemas, entre otros. }\end{array}$ \\
\hline
\end{tabular}

Tabla 2 Evolución de la didáctica de las matemáticas Fuente: Elaboración Propia

En la escuela francesa de didáctica de las matemáticas, como la llama (Artigue, 1995), hay varios elementos necesarios para modelar los fenómenos didácticos en el aprendizaje de las matemáticas, uno de esos elementos es el contrato didáctico. Un alumno construye su conocimiento matemático como resultado del proceso de resolución de problemas diseñados y propuestos por el profesor. Esto implica que tanto el profesor como el alumno esperan ciertos compromisos u obligaciones uno del otro: el alumno espera que el profesor proponga problemas alcanzables y que dirijan la didáctica hacia los temas establecidos en el programa académico, por otro lado, el profesor espera que el alumno realice las actividades que se le proponen (Gascón, 2006).

\section{Modelo educativo Integral y Flexible (MEIF)}

Hoy en día la educación en cualquiera de sus niveles ha de asumir el reto y la responsabilidad de una educación integral que no fragmente al individuo, una educación que no sólo atienda a los conocimientos sino a la pertinencia y transferencia de los conocimientos; una educación que rebase el plano del conocer para arribar el plano del hacer y del ser; educación que permita a cada persona descubrir, despertar e incrementar sus posibilidades creativas con y a través de esos conocimientos contribuyendo con esto a la realización de la persona en su totalidad y con ello al desarrollo
El Modelo Educativo Integral y Flexible (MEIF) es el modelo educativo de la UV y se caracteriza por ser:
- Integral al promover la preparación intelectual, profesional, social y humana, a través de las áreas de formación del plan de estudios.
Flexible en tiempos, espacios y contenidos ya que, dentro de ciertos límites, le permite al estudiante adecuar su trayectoria escolar a sus necesidades e intereses.

estudiante podrá:

Esta flexibilidad significa que el

Determinar su tiempo de permanencia en el Programa educativo, a través de las trayectorias escolares: mínima, promedio o máxima, con base en las estrategias de operación de su Plan de estudios (Tiempo).

- Cursar experiencias educativas (EE), incluidas en la oferta, en distintas facultades, institutos o dependencias, así como acreditarlas en diferentes modalidades de aprendizaje: presencial, semipresencial, virtual, mixta, autoaprendizaje y demostración de competencias (Espacio).

- Seleccionar las EE que cursará en cada periodo escolar con base en la trayectoria escolar elegida: mínima, promedio o máxima (para ello debe conocer el total de créditos para obtener el grado de licenciatura y el mínimo y máximo de créditos por periodo escolar), oferta de $\mathrm{EE}$, área de formación a la que pertenecen y sus características, es decir, si es obligatoria, optativa o de elección libre, así como los requisitos de las mismas (contenidos).

Con este enfoque se pretende dar paso a una enseñanza en que se armonicen los contenidos teóricos con los procedimentalesheurísticos y actitudinales. Educación que no solo se preocupe por cómo enseñar sino también por cómo aprender, es decir una enseñanza centrada en el aprendizaje, no sólo de conocimientos, sino de desarrollo de habilidades $y$ actitudes que implican una serie de aprendizajes para la vida, mismos que se corresponden con la noción de competencia. Esto es una enseñanza centrada en el estudiante. 


\section{Marco Contextual}

Esta investigación se llevó a cabo en una de las facultades de una universidad del estado de Veracruz, (desde este momento denominada UV), cabe señalar que esta institución cuenta con distintas sedes a lo largo del estado de Veracruz y se seleccionó la Facultad de Ingeniería Química de la sede Poza Rica-Tuxpan para el caso de estudio. Los grupos de nuevo ingreso del programa de Ingeniería Química en el campus Poza Rica-Tuxpan de la UV, son muy numerosos. Se abren plazas para 120 alumnos que se dividen en dos grupos de 60 alumnos cada uno. Obviamente, esto implica que los profesores no puedan atender oportunamente las demandas de atención individual de sus estudiantes. Se ha solicitado que haya más grupos pero no hay presupuesto para esa ampliación ya que se requiere infraestructura y más contratación de más profesores. Hay que recalcar que no se cuenta con un departamento de matemáticas o profesores adjuntos que puedan cubrir las necesidades de asesoría de los estudiantes.

Se han intentado establecer seminarios de matemáticas pero no se han planificado adecuadamente ya que no había material preparado con anterioridad, la asistencia no era constante, el alumno asistía sólo a aclarar las dudas del ejercicio en turno y no lo hacía para aprender, no se hizo una programación constante, no hubo personal ni recursos económicos disponibles para llevar la tarea.

\section{Metodología}

La propuesta de solución planteó un estudio basado en 3 etapas que incluyen información bibliográfica, documental y estadística, para la parte de intervención, enmarcado dentro de la modalidad investigación cuantitativa de campo de tipo descriptivo, con características de observación participante que siguió con una propuesta de un modelo didáctico para mejorar el aprendizaje en Matemáticas Básicas y que finalmente se evaluó para determinar su efectividad.

La población fue de dos grupos de nuevo ingreso, del ciclo Agosto 2017 a Enero 2018, en el programa educativo de Ingeniería Química en la Facultad de Ciencias Químicas Poza Rica, que cursan la experiencia educativa de Matemáticas Básicas.
Para tal efecto se consideraron 84 alumnos de nuevo ingreso, turno vespertino, divididos en dos grupos, el IQ-2-I e IQ-3-I, considerando uno para control (34 alumnos) y el otro de intervención (50 alumnos).

Se hizo uso de los siguientes instrumentos: Cuestionario, observación participante y entrevista. La finalidad del primero es establecer las diferencias en cuanto a habilidades para resolver problemas $\mathrm{y}$ conocimientos en los alumnos de nuevo ingreso respecto a Matemáticas Básicas, además de conocer los hábitos de estudio y actitudes que muestran en cuanto al aprendizaje en esta asignatura, tanto al inicio del estudio como después del modelo de aprendizaje propuesto. Con el segundo instrumento se registró su comportamiento dentro de la clase, tanto con el profesor como con sus pares para cruzar la información con el cuestionario, en base a una lista de cotejo para establecer el aprendizaje significativo y una guía de observación. Al final, con la entrevista se intentó definir si fue determinante el carácter y dominio de terminología para su aprendizaje en matemáticas.

La indagación bibliográfica permitió definir los indicadores y su valoración; en el cuestionario, para la parte de conocimientos se establecieron dos niveles, conocimientos básicos, cuya valoración fue correcto o incorrecto y habilidad para resolver problemas, con existe o no existe. En cuanto a la parte de actitudes se consideró una escala de muy de acuerdo, de acuerdo, en desacuerdo, muy en desacuerdo; para hábitos de estudio fue la frecuencia de realización de diversas actividades inherentes, desde siempre, la mayoría de las veces, en ocasiones y nunca.

Para la lista de cotejo se dividieron las preguntas en base a la siguiente clasificación: actividades, construcción, cooperación, autenticidad e intencionalidad, jerarquizando con mucho, poco o nada. Por último, se consideraron los parámetros de información, participación, técnica y "tiempo muerto" para la guía de observación en clase, contabilizando los momentos en que aparecen las categorías enumeradas y en la entrevista se definieron cuatro preguntas abiertas que indicaron la pertinencia del indicador relativo a terminología. 


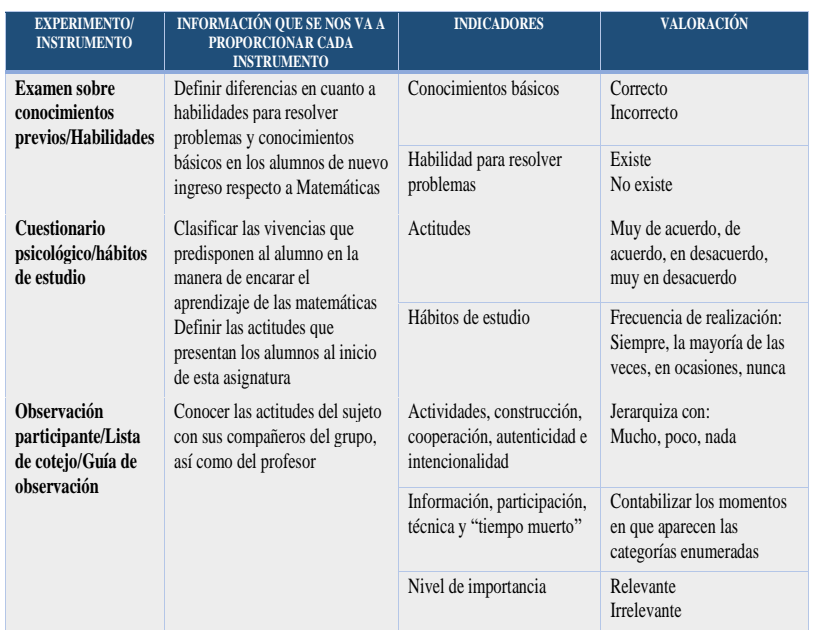

Figura 3 Resumen metodológico de la investigación. Fuente: Elaboración Propia

\section{Resultados}

El trabajo de investigación se realizó durante el periodo comprendido de Agosto 2017 Enero 2018, con un grupo control de 34 alumnos y otro de intervención de 50 alumnos. A continuación se muestran los resultados obtenidos después de realizar las actividades de intervención.

Experimento 1 Conocimientos básicos y habilidad para resolver problemas: Se aplicó un cuestionario a la muestra de estudiantes elegida, para determinar sus conocimientos básicos y su habilidad para resolver problemas en el área de matemáticas básicas.

Las respuestas obtenidas de la prueba de habilidad para resolver problemas se muestran en la tabla 2 y en el gráfico 1.

\begin{tabular}{|c|c|c|c|c|c|c|}
\hline \multirow{2}{*}{ Pregunta } & \multicolumn{3}{|c|}{ Grupo control } & \multicolumn{3}{|c|}{$\begin{array}{l}\text { Grupo } \\
\text { intervención }\end{array}$} \\
\hline & $\mathrm{RC}$ & RI & $\%$ RC & $\mathrm{RC}$ & RI & $\% R C$ \\
\hline $\begin{array}{ll}\text { Relacionar } & \text { datos con } \\
\text { una } & \text { expresión } \\
\text { algebraica } & \\
\end{array}$ & 17 & 17 & 50 & 22 & 28 & 44 \\
\hline $\begin{array}{l}\text { Elección de envase en } \\
\text { función de volumen }\end{array}$ & 20 & 14 & 59 & 31 & 19 & 62 \\
\hline $\begin{array}{l}\text { Justificación de toma } \\
\text { de decisión }\end{array}$ & 22 & 12 & 65 & 29 & 21 & 58 \\
\hline Razón y semejanza & 29 & 5 & 85 & 43 & 7 & 86 \\
\hline $\begin{array}{l}\text { Solución de problema } \\
\text { de temperatura }\end{array}$ & 30 & 4 & 88 & 42 & 8 & 84 \\
\hline Promedio: & & & 69 & & & 67 \\
\hline
\end{tabular}

Tabla 2 Respuestas obtenidas con la prueba de habilidad para resolver problemas

Fuente: Elaboración Propia

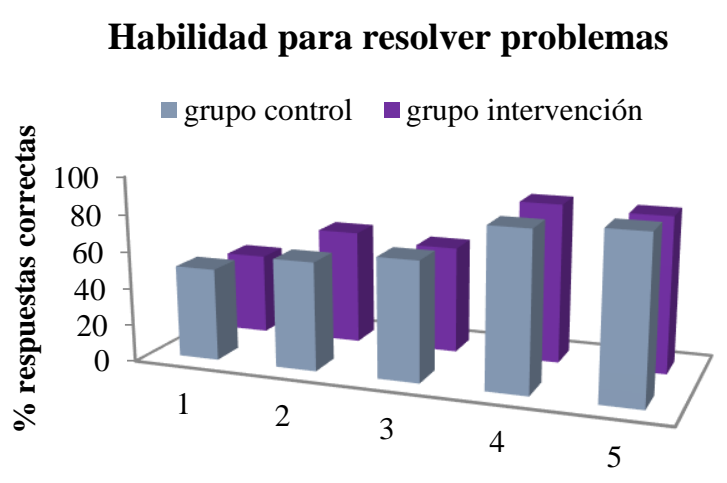

No. Pregunta

Gráfico 1 Habilidad para resolver problemas en los alumnos de Ingeniería Química, región Poza Rica, que cursaron matemáticas básicas en el periodo agosto 2017 febrero 2018

Fuente: Elaboración Propia

Las respuestas obtenidas sobre conocimientos básicos se muestran en la tabla 3 y el gráfico 2 .

\begin{tabular}{|l|c|c|c|c|c|c|}
\hline \multicolumn{1}{|c}{ Pregunta } & RC control & \multicolumn{3}{c|}{$\begin{array}{c}\text { Grupo } \\
\text { intervención }\end{array}$} \\
\hline $\begin{array}{l}\text { Reducción de término } \\
\text { común con fracciones }\end{array}$ & 8 & 26 & 24 & 10 & 40 & 20 \\
\hline $\begin{array}{l}\text { Factorizar ecuación } \\
\text { de 2. grado }\end{array}$ & 28 & 6 & 82 & 36 & 14 & 72 \\
\hline $\begin{array}{l}\text { Simplificar una } \\
\text { expresión algebraica } \\
\text { racional }\end{array}$ & 10 & 24 & 29 & 15 & 35 & 30 \\
\hline $\begin{array}{l}\text { Encontrar las raíces } \\
\text { de una ecuación de } \\
\text { segundo grado }\end{array}$ & 9 & 25 & 26 & 22 & 28 & 44 \\
\hline $\begin{array}{l}\text { Resolver un sistema } \\
\text { de dos ecuaciones } \\
\text { lineales con dos } \\
\text { incógnitas }\end{array}$ & 31 & 3 & 91 & 46 & 4 & 92 \\
\hline Promedio: & & & & & & \\
\hline
\end{tabular}

Tabla 3 Respuestas obtenidas con las pruebas de conocimientos básicos

Fuente: Elaboración Propia

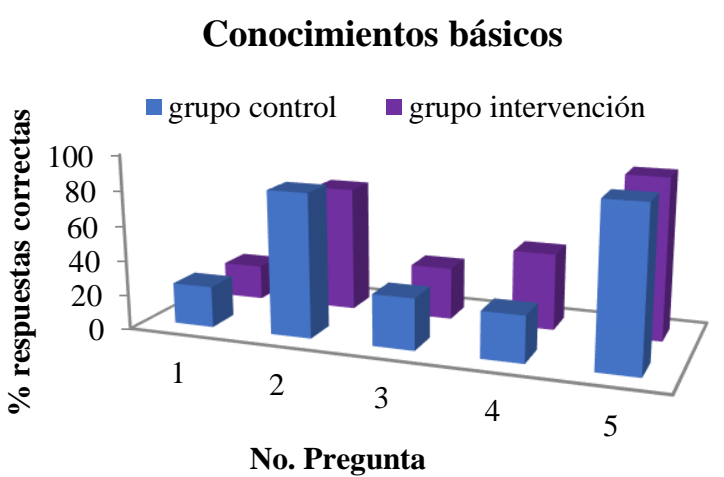

Gráfico 2 Conocimientos básicos en los alumnos de Ingeniería Química, región Poza Rica, que cursaron matemáticas básicas en el periodo agosto 2017-febrero 2018

Fuente: Elaboración Propia 
Al efectuar un análisis de los valores obtenidos con los test de habilidad para resolver problemas y conocimientos generales se observó que en general, ambos grupos poseen la habilidad para resolver problemas en forma aceptable a diferencia de los conocimientos básicos en el cual obtuvieron calificación reprobatoria.

Se ofrece un resumen de estos resultados en la tabla 4.

\begin{tabular}{|c|c|c|}
\hline \multirow[b]{2}{*}{ Tipo de prueba } & \multicolumn{2}{|c|}{ Promedio obtenido } \\
\hline & $\begin{array}{l}\text { Grupo } \\
\text { control }\end{array}$ & $\begin{array}{c}\text { Grupo de } \\
\text { intervención }\end{array}$ \\
\hline $\begin{array}{l}\text { Habilidad para resolver } \\
\text { problemas }\end{array}$ & 69 & 67 \\
\hline Conocimientos básicos & 51 & 52 \\
\hline
\end{tabular}

Tabla 4 Resultados al inicio de la investigación Fuente: Elaboración Propia

Experimento 2 Hábitos de estudio: El instrumento consta de doce preguntas y cuatro categorías en función de la frecuencia en las actividades: siempre, regularmente, raras veces y nunca.

En la tabla 5 se muestran los cuestionamientos. Cada uno de ellos se tomó de la encuesta que realiza el departamento de Tutorías de la UV. Se inicia con la frase: Frecuencia con que realizas las siguientes actividades en la clase de matemáticas básicas.

\begin{tabular}{|l|l|}
\hline 1 & Escuchar a tu maestro explicar la clase \\
\hline 2 & Realizar ejercicios en forma individual \\
\hline 3 & Resolver problemas en pequeños grupos \\
\hline 4 & Traer a la mano objetos como regla o calculadora \\
\hline 5 & Realizar un test o examen \\
\hline 6 & Asistir a clases regularmente \\
\hline 7 & Asistir puntualmente a clases \\
\hline 8 & Tomar apuntes \\
\hline 9 & Realizar preguntas en clase para aclarar tus dudas \\
\hline 10 & $\begin{array}{l}\text { Discutir los puntos de vista del maestro o de mis } \\
\text { compañeros }\end{array}$ \\
\hline 11 & Estudiar con anticipación para los exámenes \\
\hline 12 & $\begin{array}{l}\text { Consultar bibliografía o materiales de lectura para } \\
\text { reforzar lo visto en clase. }\end{array}$ \\
\hline
\end{tabular}

Tabla 5 Listado de preguntas en relación a los hábitos de estudio

Fuente: Elaboración Propia

A continuación, en la tabla 6 se muestran los resultados de la evaluación inicial tanto en el grupo control como de intervención.

\begin{tabular}{|r|r|r|r|r|r|r|r|r|}
\hline & \multicolumn{4}{|c}{ GRUPO CONTROL } & \multicolumn{3}{c|}{ GRUPO DE INTERVENCIÓN } \\
\hline P & Siempre & Regular & $\begin{array}{l}\text { Raras } \\
\text { Veces }\end{array}$ & Nunca & Siempre & Regular & $\begin{array}{r}\text { Raras } \\
\text { Veces }\end{array}$ & Nunca \\
\hline $\mathbf{1}$ & 18 & 82 & 0 & 0 & 18 & 76 & 6 & 0 \\
\hline $\mathbf{2}$ & 24 & 71 & 6 & 0 & 12 & 66 & 22 & 0 \\
\hline $\mathbf{3}$ & 0 & 24 & 76 & 0 & 2 & 28 & 70 & 0 \\
\hline $\mathbf{4}$ & 41 & 41 & 18 & 0 & 42 & 46 & 12 & 0 \\
\hline $\mathbf{5}$ & 29 & 29 & 29 & 12 & 28 & 30 & 30 & 12 \\
\hline $\mathbf{6}$ & 82 & 18 & 0 & 0 & 70 & 18 & 12 & 0 \\
\hline $\mathbf{7}$ & 71 & 29 & 0 & 0 & 64 & 30 & 6 & 0 \\
\hline $\mathbf{8}$ & 71 & 29 & 0 & 0 & 76 & 24 & 0 & 0 \\
\hline $\mathbf{9}$ & 12 & 35 & 47 & 6 & 12 & 36 & 46 & 6 \\
\hline $\mathbf{1 0}$ & 12 & 29 & 41 & 18 & 12 & 26 & 50 & 12 \\
\hline $\mathbf{1 1}$ & 24 & 59 & 18 & 0 & 24 & 62 & 14 & 0 \\
\hline 12 & 12 & 18 & 59 & 12 & 18 & 12 & 59 & 12 \\
\hline
\end{tabular}

Tabla 6 Porcentaje de frecuencias obtenidas al aplicar la encuesta sobre hábitos de estudio, al inicio del periodo Fuente: Elaboración Propia

Comparando el promedio por alumno de respuestas favorables (siempre y regularmente), representada por $\mathrm{F}$ contra las no favorables raras veces y nunca), caracterizada por las letras NF, se llega a los datos de la tabla 7.

\begin{tabular}{|c|c|c|c|c|}
\hline \multirow{2}{*}{ Valor } & \multicolumn{2}{|c|}{$\begin{array}{c}\text { Grupo de } \\
\text { control }\end{array}$} & \multicolumn{2}{c|}{$\begin{array}{c}\text { Grupo } \\
\text { intervención }\end{array}$} \\
& F & NF & F & NF \\
\hline Promedio/alumnos & 8.59 & 3.41 & 8.28 & 3.27 \\
\hline
\end{tabular}

Tabla 7 Resultados obtenidos al inicio del periodo tanto en el grupo control como el de intervención Fuente: Elaboración Propia

Se puede observar que hay una buena tendencia hacia las frecuencias de siempre y regularmente tanto en el grupo control como en el de intervención.

Se hizo un comparativo en cuanto a número promedio de respuestas de más frecuencia y se determinó un valor muy semejante en el promedio por alumno y por lo tanto es válido el estudio.

Experimento 3 Actitud frente a las matemáticas (guía de observación): La actitud ante las matemáticas se considera un factor que afecta al aprendizaje. El hecho de inferir conclusiones de la actitud presenta dificultades por ser ésta una componente afectiva.

Para este experimento se diseñó un sistema de categorías de interacción didáctica, de contenido matemático y de comprensión de contenido, que fueron analizadas. 
El equipo se conformó por un docente, un alumno sobresaliente y varios pupilos que quisieron participar a través de los datos recogidos en diversas fuentes (cuadernos de alumnos, tareas de los alumnos, pruebas de evaluación, guía de observación y cuestionario de actitudes). La validez y fiabilidad de la investigación está en función de lo observado, registrado y la experiencia del docente en la enseñanza de las matemáticas. Los aspectos a considerar son la búsqueda de posibles causas socio-afectivas, curriculares y de opinión/actitud de estos alumnos hacia las matemáticas que originan dificultades en la enseñanza/aprendizaje de las mismas. Se requirió una planificación de las sesiones, un análisis del contenido, estrategias para su organización y presentación en el aula, y un procedimiento de observación.

A la vista de los resultados se resume lo siguiente:

- Los alumnos conceden una mayor importancia a las matemáticas de cara a un futuro y menos en su formación actual.

- $\quad$ El agrado de los alumnos por esta materia dentro de su Currículo es medio.

En general, dedican poco tiempo al estudio de las matemáticas.

La valoración de su actitud hacia las matemáticas, en una escala de 0 a 10 , está entorno al 5.

- El método de estudio y las propias limitaciones para la comprensión de las matemáticas son las principales causas internas de su actitud hacia esta disciplina.

- Los alumnos señalan, que las limitaciones personales para la comprensión de las matemáticas son la causa que más influye. La carencia de conocimientos previos y el método de estudio aparecen, ambos con igual frecuencia, en segundo lugar.

En cuanto a las creencias acerca de la naturaleza de las matemáticas y de su enseñanza y aprendizaje respecta, los estudiantes parecen considerar las matemáticas como útiles y necesarias tanto para desenvolverse adecuadamente en la sociedad como para asimilar y dominar otras asignaturas que guardan relación con dicha disciplina. De esta forma quedaría patente el deseo de dominio en la materia.
Experimento 4 Actitud frente a las matemáticas (cuestionario): Con este instrumento se pretendió conocer la forma en que el alumno se percibe frente a un problema matemático o simplemente frente a una clase de esta temática. En cierto modo se esperó que reflejara su autoestima o las posibles situaciones "incomodas" o "traumáticas" que ha experimentado a lo largo de su trayectoria académica. El instrumento consta de 24 preguntas y cuatro categorías en función de la preferencia o concordancia con su manera de percibir las matemáticas: muy de acuerdo, de acuerdo, en desacuerdo, muy en desacuerdo. (Brousseau, 2007). El cuestionario base de este estudio se tomó parcialmente de (Caballero, 2007), Las actitudes y emociones ante las Matemáticas. En la tabla 8 se muestra un listado de las preguntas incluidas.

\begin{tabular}{|c|c|}
\hline 1 & $\begin{array}{l}\text { es se me hacen eternas, son muy pesadas, no estoy a gusto } \\
\text { deseos de salir corriendo }\end{array}$ \\
\hline 2 & $\begin{array}{l}\text { Disfruto los días que no tenemos clases porque no me interesan ni } \\
\text { me atraen }\end{array}$ \\
\hline 3 & Ante un problema complicado suelo darme por vencido fácilmente \\
\hline 4 & $\begin{array}{l}\text { Cuando me enfrento a un problema experimento mucha curiosidad } \\
\text { por conocer la solución }\end{array}$ \\
\hline 5 & $\begin{array}{l}\text { Me angustio y siento miedo cuando el profesor me propone «por } \\
\text { sorpresa» que resuelva un problema }\end{array}$ \\
\hline 6 & $\begin{array}{l}\text { Cuando resuelvo problemas en grupo tengo más seguridad en mí } \\
\text { mismo }\end{array}$ \\
\hline 7 & $\begin{array}{l}\text { ión de un problema } \\
\text { ervioso }\end{array}$ \\
\hline 8 & $\begin{array}{l}\text { un problema tengo la sensación de } \\
\text { dido el tiempo }\end{array}$ \\
\hline 9 & $\begin{array}{l}\text { Me provoca gran satisfacción llegar a resolver con éxito un } \\
\text { problema }\end{array}$ \\
\hline 10 & $\begin{array}{l}\text { uando fracasan mis intentos por resolver un problema lo intento } \\
\text { e nuevo }\end{array}$ \\
\hline 11 & ma exige esfuerzo, perseverancia y \\
\hline 12 & nbitos de la \\
\hline 13 & uir un buen \\
\hline 14 & temáticas es tan importante \\
\hline 15 & $\begin{array}{l}\text { Si no se comprenden las matemáticas, difícilmente se podrán } \\
\text { asimilar y dominar otras asignaturas relacionadas con ella (como } \\
\text { física, química, etc.) }\end{array}$ \\
\hline 16 & les, aburridas y alejadas de la realidad \\
\hline 17 & $\begin{array}{l}\text { es fundamental aprenderse de memoria los } \\
\text { las y reglas }\end{array}$ \\
\hline 18 & problema es más importante el resultado \\
\hline 19 & matemáticas es a través del estudio \\
\hline 20 & $\begin{array}{l}\text { Cuando resuelvo un problema suelo dudar de si el resultado es } \\
\text { correcto }\end{array}$ \\
\hline 21 & $\begin{array}{l}\text { Tengo confianza en mí mismo cuando me enfrento a los problemas } \\
\text { de matemáticas }\end{array}$ \\
\hline 22 & huy capa \\
\hline 23 & \begin{tabular}{|l}
$\begin{array}{l}\text { Estoy calmado y tranquilo cuando resuelvo problemas de } \\
\text { matemáticas }\end{array}$ \\
\end{tabular} \\
\hline 24 & $\begin{array}{l}\text { El gusto por las matemáticas me influyó a la hora de escoger una } \\
\text { determinada modalidad de bachillerato }\end{array}$ \\
\hline
\end{tabular}

Tabla 8 Listado de preguntas sobre las actitudes respecto a las matemáticas 
Los resultados de la evaluación inicial tanto en el grupo control como de intervención se muestran en la tabla 9. Las celdas sombreadas representan las respuestas que se identificarían como resultado de una actitud positiva hacia las matemáticas, la tabla 10 muestra un resumen de ellas.

\begin{tabular}{|c|c|c|c|c|c|c|c|c|}
\hline Preg & \multicolumn{9}{|c}{ G. CONTROL } & \multicolumn{4}{c|}{ G. INTERVENCIÓN } \\
\hline $\mathbf{1}$ & 0 & 10 & $\mathbf{2 0}$ & $\mathbf{4}$ & 3 & 15 & $\mathbf{1 9}$ & $\mathbf{1 3}$ \\
\hline $\mathbf{2}$ & 0 & 5 & $\mathbf{2 5}$ & $\mathbf{4}$ & 0 & 6 & $\mathbf{3 5}$ & $\mathbf{9}$ \\
\hline $\mathbf{3}$ & 0 & 1 & $\mathbf{2 4}$ & $\mathbf{9}$ & 0 & 6 & $\mathbf{3 4}$ & $\mathbf{1 0}$ \\
\hline $\mathbf{4}$ & $\mathbf{1 4}$ & $\mathbf{1 7}$ & 3 & 0 & $\mathbf{1 7}$ & $\mathbf{2 7}$ & 6 & 0 \\
\hline $\mathbf{5}$ & 3 & 8 & $\mathbf{1 8}$ & $\mathbf{5}$ & 8 & 23 & $\mathbf{1 6}$ & $\mathbf{3}$ \\
\hline $\mathbf{6}$ & 14 & 14 & $\mathbf{6}$ & $\mathbf{0}$ & 21 & 20 & $\mathbf{9}$ & $\mathbf{0}$ \\
\hline $\mathbf{7}$ & 5 & 7 & $\mathbf{2 0}$ & $\mathbf{2}$ & 6 & 12 & $\mathbf{2 9}$ & $\mathbf{3}$ \\
\hline $\mathbf{8}$ & 2 & 8 & $\mathbf{2 1}$ & $\mathbf{3}$ & 0 & 12 & $\mathbf{3 1}$ & $\mathbf{7}$ \\
\hline $\mathbf{9}$ & $\mathbf{2 5}$ & $\mathbf{9}$ & 0 & 0 & $\mathbf{3 5}$ & $\mathbf{9}$ & 4 & 2 \\
\hline $\mathbf{1 0}$ & $\mathbf{1 0}$ & $\mathbf{2 0}$ & 4 & 0 & $\mathbf{1 5}$ & $\mathbf{2 9}$ & 6 & 0 \\
\hline $\mathbf{1 1}$ & $\mathbf{2 5}$ & $\mathbf{5}$ & 3 & 1 & $\mathbf{3 6}$ & $\mathbf{5}$ & 6 & 3 \\
\hline $\mathbf{1 2}$ & $\mathbf{2 2}$ & $\mathbf{7}$ & 5 & 0 & $\mathbf{3 0}$ & $\mathbf{1 5}$ & 5 & 0 \\
\hline $\mathbf{1 3}$ & $\mathbf{2 0}$ & $\mathbf{1 2}$ & 2 & 0 & $\mathbf{3 1}$ & $\mathbf{1 9}$ & 0 & 0 \\
\hline $\mathbf{1 4}$ & $\mathbf{2 7}$ & $\mathbf{7}$ & 0 & 0 & $\mathbf{4 0}$ & $\mathbf{8}$ & 2 & 0 \\
\hline $\mathbf{1 5}$ & $\mathbf{2 0}$ & $\mathbf{1 2}$ & 2 & 0 & $\mathbf{3 2}$ & $\mathbf{1 5}$ & 0 & 3 \\
\hline $\mathbf{1 6}$ & 0 & 5 & $\mathbf{2 0}$ & $\mathbf{9}$ & 3 & 0 & $\mathbf{3 7}$ & $\mathbf{1 0}$ \\
\hline $\mathbf{1 7}$ & $\mathbf{4}$ & $\mathbf{9}$ & 16 & 5 & $\mathbf{6}$ & $\mathbf{1 5}$ & 23 & 6 \\
\hline $\mathbf{1 8}$ & 2 & 11 & $\mathbf{2 1}$ & $\mathbf{0}$ & 4 & 15 & $\mathbf{3 0}$ & $\mathbf{1}$ \\
\hline $\mathbf{1 9}$ & 3 & 11 & $\mathbf{2 0}$ & $\mathbf{0}$ & 3 & 16 & $\mathbf{3 1}$ & $\mathbf{0}$ \\
\hline $\mathbf{2 0}$ & 4 & 14 & $\mathbf{1 6}$ & $\mathbf{0}$ & 6 & 21 & $\mathbf{2 3}$ & $\mathbf{0}$ \\
\hline $\mathbf{2 1}$ & $\mathbf{4}$ & $\mathbf{2 3}$ & 7 & 0 & $\mathbf{7}$ & $\mathbf{2 8}$ & 12 & 3 \\
\hline $\mathbf{2 2}$ & $\mathbf{3}$ & $\mathbf{2 1}$ & 10 & 0 & $\mathbf{3}$ & $\mathbf{3 2}$ & 11 & 4 \\
\hline $\mathbf{2 3}$ & $\mathbf{4}$ & $\mathbf{2 4}$ & 6 & 0 & $\mathbf{6}$ & $\mathbf{3 6}$ & 8 & 0 \\
\hline $\mathbf{2 4}$ & $\mathbf{7}$ & $\mathbf{1 3}$ & 12 & 2 & $\mathbf{1 0}$ & $\mathbf{2 0}$ & 18 & 2 \\
\hline Total & $\mathbf{1 8 5}$ & $\mathbf{1 7 9}$ & $\mathbf{2 1 1}$ & $\mathbf{3 6}$ & $\mathbf{2 6 8}$ & $\mathbf{2 5 8}$ & $\mathbf{2 9 4}$ & $\mathbf{5 6}$ \\
\hline & & & & & & & & \\
\hline
\end{tabular}

Tabla 9 Resultados obtenidos tanto en el grupo control como el de intervención al inicio del periodo

Fuente: Elaboración Propia

\begin{tabular}{|l|l|}
\hline \multicolumn{1}{|c|}{$\begin{array}{c}\text { Respuesta } \\
\text { indicativa de } \\
\text { actitud positiva }\end{array}$} & \multicolumn{1}{|c|}{$\begin{array}{c}\text { Preguntas que se identifican } \\
\text { con esa respuesta }\end{array}$} \\
\hline $\begin{array}{l}\text { En desacuerdo, muy } \\
\text { en desacuerdo }\end{array}$ & $2,3,5,6,7,8,16,18,19$ y 20 \\
\hline $\begin{array}{l}\text { Muy de acuerdo, de } \\
\text { acuerdo }\end{array}$ & $\begin{array}{l}4,9,10,11,12,13,14,15,17, \\
21,22 \text { y } 23\end{array}$ \\
\hline
\end{tabular}

Tabla 10 Resumen de las preguntas que mostrarían una actitud positiva en base a su respuesta.

Fuente: Elaboración Propia

Las respuestas esperadas ante una actitud negativa hacia las matemáticas. Se muestra un listado en la tabla 11.

\begin{tabular}{|l|l|}
\hline \multicolumn{1}{|c}{$\begin{array}{c}\text { Respuesta } \\
\text { indicativa de } \\
\text { actitud negativa }\end{array}$} & \multicolumn{1}{c|}{$\begin{array}{c}\text { Preguntas que se identifican } \\
\text { con esa respuesta }\end{array}$} \\
\hline $\begin{array}{l}\text { Muy de acuerdo, de } \\
\text { acuerdo }\end{array}$ & $2,3,5,6,7,8,16,18,19$ y 20 \\
\hline $\begin{array}{l}\text { En desacuerdo, muy } \\
\text { en desacuerdo }\end{array}$ & $\begin{array}{l}4,9,10,11,12,13,14,15,17, \\
21,22 \text { y } 23\end{array}$ \\
\hline
\end{tabular}

Tabla 11 Resumen de las preguntas que mostrarían una actitud negativa en base a su respuesta

Fuente: Elaboración Propia
En base a lo anterior, se efectuó la sumatoria de las áreas sombreadas para determinar la actitud hacia las matemáticas tanto en el grupo control como en el de intervención. Los resultados obtenidos se resumen en la tabla 12.

\begin{tabular}{|l|c|c|c|c|c|}
\hline \multicolumn{1}{|c}{ Valor } & \multicolumn{4}{c}{ Promedio/alumnos } & \multicolumn{1}{c|}{ Prom } \\
& MA & A & D & MD & final \\
\hline Grupo control & 5.44 & 5.26 & 6.21 & 1.06 & $\mathbf{4 . 4 9}$ \\
\hline $\begin{array}{l}\text { Grupo.de } \\
\text { intervención }\end{array}$ & 5.36 & 5.16 & 5.88 & 1.12 & $\mathbf{4 . 3 8}$ \\
\hline
\end{tabular}

Tabla 12 Resultados obtenidos al inicio del periodo tanto en el grupo control como el de intervención Fuente: Elaboración Propia

Se puede observar que el valor del promedio final tanto en el grupo de control como en el de intervención tienen valores muy semejantes (4.49 y 4.38), por lo tanto es válido el estudio.

Después de implementar el modelo didáctico y evaluarlo se comprobó que se disminuyen los índices de reprobación del área de matemáticas básicas en la carrera de Ingeniería Química. Los gráficos 3 y 4 muestran estos resultados.

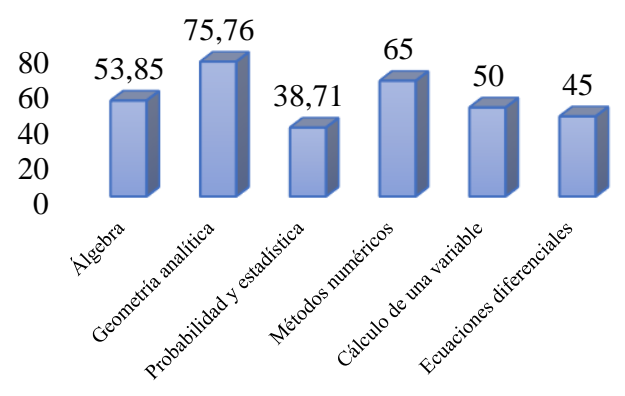

Gráfico 3 Índice de reprobación del periodo Febrero-Julio 2017, de la carrera de Ingeniería Química Fuente: Elaboración Propia

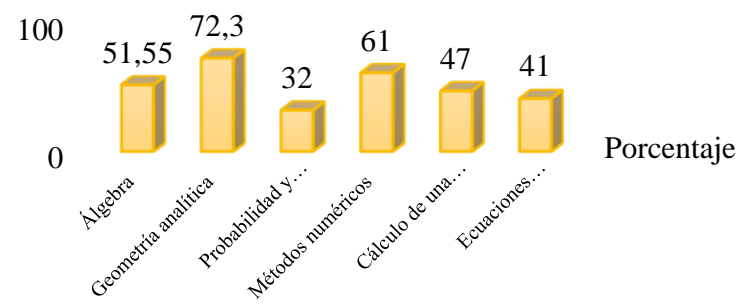

Gráfico 4 Índice de reprobación del periodo Agosto 2017 Enero 2018, de la carrera de Ingeniería Química Fuente: Elaboración Propia 


\section{Conclusiones}

El modelo propuesto permitió observar algunos cambios significativos en referencia a los hábitos de estudio y actitud que presentan los alumnos frente a las matemáticas. La manifiesta mejora, en general, de los hábitos de estudio hacia las matemáticas, como tomar notas, consultar bibliografía y realizar preguntas para aclarar dudas, demuestra que si el alumno tiene apoyos didácticos, asesorías, material a su disposición y sobre todo toma conciencia de su realidad, puede lograr una autonomía de su aprendizaje.

El trabajo de investigación se enfocó en determinar las mejores estrategias de aprendizaje que debe desarrollar el estudiante de matemáticas básicas del programa de Ingeniería Química con la finalidad de lograr un aprendizaje significativo manifestado en la resolución de problemas matemáticos.

Con la puesta en marcha del modelo didáctico se logró mejorar la atención por parte de los estudiantes, un incemento en su motivación por aprender, muchos de ellos potenciaron sus capacidades autodidáctas, en lo emocional, rompió con muchas barreras por lo que la mayoría de los estudiantes del grupo muestra externan de manera clara sus dudas y son de gran apoyo para motivar a sus profesores para el desarrollo de estrategias de enseñanza adecuadas al nivel de conocimiento y aprendizaje que reflejan un mayor aprovechamiento.

Finalmente, la aplicación de estrategias didácticas permitó una reducción en los índices de reprobación así como la aplicación adecuada de un modelo educativo competitivo.

\section{Referencias}

Artigue, M. (1995). Epistémologie et didactique. Rechseches en Didatique des Mathématiques, 241-286.

Brousseau, G. (2007). La théorie des situations didactiques. Genoble, France: La Pensée Sauvage.

Caballero, L. J. (2007). Las actitudes y emociones ante las Matemáticas de los estudiantes para Maestros de la Facultad de Educación de la Universidad de Extremadura.
Gascón, Y. C. (2006). Estudiar matemáticas. El eslabón perdido entre la enseñanza y el aprendizaje. Barcelona: Horsori.

Ruiz, A. V. (2006). Epistemología y construcción de una nueva disciplina científica: la Didactique des mathématiques. UNICIENCIA, 20(2). 